\title{
Microstructure and impedance spectroscopy of 3YTZP/SWNT ceramic nanocomposites
}

\author{
R. Poyato ${ }^{1, *}$, J. Macías-Delgado ${ }^{1,2}$, A. Gallardo-López ${ }^{1,2}$, A. Muñoz ${ }^{2}$ and
}

A. Domínguez-Rodríguez ${ }^{2}$.

1. Inst. Ciencia de Materiales de Sevilla (CSIC-Univ. Sevilla), Avda. Américo Vespucio 49, 41092 Sevilla, Spain

2. Dept. Física de la Materia Condensada, Universidad de Sevilla, apdo. 1065, 41080 Sevilla, Spain

\begin{abstract}
This work provides new insights on microstructure and electrical properties of $3 \mathrm{~mol} \%$ $\mathrm{Y}_{2} \mathrm{O}_{3}-\mathrm{ZrO}_{2}$ (3YTZP) composites with 0.5 , 1 , and 1.5 vol\% single walled carbon nanotubes (SWNTs). The composites were spark plasma sintered (SPS) in identical conditions at $1250{ }^{\circ} \mathrm{C}$ from powder prepared by two different processing routines, with the aim of optimizing the SWNTs dispersion throughout the ceramic matrix. High densification and submicrometric grain size were achieved in all the composites. Electrical properties of the composites were characterized in a wide temperature range, and modelling of the impedance properties was approached by means of an equivalent circuit that allows separation of the individual SWNT bundles contribution to resistance from the resistance due to junctions between bundles. Effects of the homogeneous distribution of SWNTs at the ceramic grain boundaries on the crystalline phases, percolation threshold, total conductivity and evolution of junctions' resistivity with temperature were analysed and discussed.
\end{abstract}

Keywords: Composites; Carbon nanotubes; Processing; Impedance Spectroscopy

\footnotetext{
* Corresponding author.

E-mail address: rosalia.poyato@icmse.csic.es

Phone number: +34954 489534

FAX number: +34954 460665
} 


\section{Introduction}

The exceptional mechanical behavior and outstanding multifunctional features of carbon nanotubes (high Young' modulus, high tensile strength, elevated resilience, high electrical conductivity or good thermal conductivity) have made them ideal materials to tailor ceramic composites at nanometric scale, because it was expected that some of the attractive properties of CNTs would be transferred to the resulting composites. ${ }^{1-4}$ Nowadays, the development of industry and society requires composite materials not only possessing excellent mechanical properties but also outstanding functional properties, and the study of composites with CNTs have been shown to be one of the emerging areas within the study of nanotubes, with an increase from 13 to $27 \%$ of the total publications from 2001 to $2011^{3}$

In the last years, the thermal and electrical properties of CNT ceramic matrix composites have been studied in several research papers, including thermal or electrical conductivity, ${ }^{5-7}$ thermal shock resistance, ${ }^{8}$ electrical percolation threshold ${ }^{9-13}$ or dielectric behaviour. ${ }^{9,10}$ Enhanced electrical conductivity and dielectric constant values have been reported for composites with $\mathrm{Al}_{2} \mathrm{O}_{3}{ }^{7,9,12}$ or zirconia ${ }^{10,13}$ matrix when the CNT concentration approaches to the percolation threshold.

The study of the AC electrical properties of ceramic/CNT composites has recently also come into focus ${ }^{14-17}$ and the different contributions to electrical conductivity in composites with a percolating network have been analysed. Charge transport along the CNT shells and hopping conduction across nanotube-nanotube junctions were proposed as the main contributions to conductivity in $\mathrm{Si}_{3} \mathrm{~N}_{4} /$ multi walled carbon nanotubes (MWNT) composites at room temperature. ${ }^{16}$ The study of temperature dependence of conductivity from 5 to $300 \mathrm{~K}$ carried out by Ahmad et al. ${ }^{15}$ 
for $\mathrm{Al}_{2} \mathrm{O}_{3} / \mathrm{MWNT}$ composites suggested that for temperatures higher than $50 \mathrm{~K}$, conduction can be ascribed to thermal fluctuation-induced tunneling of the charge carriers through junctions between MWNTs, while at temperatures below $50 \mathrm{~K}$, the conduction can be attributed to three dimensional variable range hopping through MWNTs network in the alumina matrix. Fluctuation-induced electron tunnelling across nanotube-nanotube junctions was also pointed as responsible for conduction in 3YTZP/SWNT composites from room temperature to $\sim 180{ }^{\circ} \mathrm{C},{ }^{17}$ whereas at higher temperatures, a mixed ionic-electronic conductivity was proposed. ${ }^{14,17}$

One of the main obstacles to the obtaining of ceramic matrix composites with optimized properties is the difficulty in achieving a homogeneous distribution of CNTs throughout the ceramic matrix. ${ }^{4}$ The tendency of CNTs to form bundles due to Van der Waals forces, and also the phase separation and demixion thermodynamically promoted when mixing thin rods (CNTs) with spheres (ceramic powder) ${ }^{12}$ inhibit the adequate dispersion of CNTs in the ceramic powder, resulting in the presence of CNT agglomerates in the composite microstructure after sintering. Although aqueous colloidal processing including CNT acid-treatment has been shown to be an efficient technique in order to obtain adequate dispersion of CNTs in ceramic matrix composites, ${ }^{18}$ the presence of agglomerates seems to be unavoidable. ${ }^{17,19,20}$ Recently, processing efforts devoted to minimize the presence of these agglomerates, including new steps in colloidal processing such as CNTs or composite powder blend freezedrying or high-energy sonication by means of ultrasonic probe, have been approached. ${ }^{12,17,21,22}$ Significant effects of the processing route on CNT dispersion and high-temperature mechanical properties have been reported for 3YTZP/SWNT composites. $^{21}$ 
Regarding the electrical properties, in a previous study ${ }^{17}$ we have shown that the presence of SWNT agglomerates leads to an overestimated value of the percolation threshold, as the real CNT content at the ceramic grain boundaries is lower than the nominal one. Thus, the high percolation thresholds reported for zirconia/CNT composites (5.5 and 3 vol\% CNTs for $3 \mathrm{YTZP} / \mathrm{MWNT}^{10,11}$ and $8 \mathrm{~mol} \% \mathrm{Y}_{2} \mathrm{O}_{3^{-}}$ $\mathrm{ZrO}_{2} / \mathrm{SWNT}^{14}$ composites, respectively) are probably consequence of the processing routine by ball milling the mixture of CNTs and ceramic powders used to prepare the composites, which results in the existence of CNT agglomerates after sintering. On the contrary, lower percolation thresholds are published when composites are prepared using processing routines specially designed to avoid CNT agglomerates ( 0.6 and 1.5 vol\% CNTs for $\mathrm{Al}_{2} \mathrm{O}_{3} / \mathrm{MWNT}^{12}$ and $3 \mathrm{YTZP} / \mathrm{SWNT}^{17}$ composites, respectively). Also, we have shown that the presence of SWNT agglomerates or high-diameter bundles results in high nanotube-nanotube junction resistivity, which will hinder the overall composite conductivity. ${ }^{17}$ In this context, the study of the effect of processing on the CNT distribution and on the electrical properties appears as a challenge. To the best of our knowledge, studies on the influence of processing routine on the electrical properties of ceramic matrix composites have not been published up to date.

In this study, $3 \mathrm{~mol} \% \mathrm{Y}_{2} \mathrm{O}_{3}-\mathrm{ZrO}_{2}$ composites with $0.5,1$ and 1.5 vol\% SWNT were spark plasma sintered from powder prepared by two different aqueous colloidal processing routines, with the aim of optimizing the SWNTs dispersion throughout the ceramic matrix and minimizing the presence of SWNT agglomerates. Microstructure and phases of the sintered materials have been characterized, as well as electrical properties by impedance spectroscopy in a wide temperature range. Percolation threshold was determined from conductivity measurements at room temperature. 
Modelling of the impedance properties allowed the analysis of the evolution of SWNT bundles and junctions' resistivity with temperature.

\section{Experimental procedure}

\subsection{Materials processing and sintering}

$3 \mathrm{YTZP} / \mathrm{SWNT}$ composites with different carbon nanotube content $(0.5,1$ and 1.5 vol\%) were prepared from $3 \mathrm{~mol} \%$ yttria stabilized tetragonal zirconia powder (3YTZP, $40 \mathrm{~nm}$ particle size and 99\% purity) supplied by Nanostructured and Amorphous Materials Inc. (Houston, TX) and HIP-co purified SWNTs provided by Carbon Solutions Inc. (Riverside, CA).

Acid treatment of the SWNTs was carried out using a mixture of concentrated sulfuric acid (98\%) and nitric acid (70\%) in the ratio 3:1, with the aim of disentangle and cut the raw SWNT ropes, as described elsewhere. ${ }^{18}$ The acid treated SWNTs were washed in high purity ethanol for several times and freeze-dried in order to avoid possible re-agglomeration. Colloidal processing of the composite powders was carried out in aqueous solution of $\mathrm{pH} 12$, where both the SWNTs and the 3YTZP nanoparticle surfaces are negatively charged, using $\mathrm{NH}_{3}$ to adjust the solution $\mathrm{pH}$. In a first step, independent SWNTs and ceramic powder solutions were prepared and subjected to ultrasonic agitation for $15 \mathrm{~min}$ in an ultrasonic bath, or by means of an ultrasonic probe (Model KT-600, Kontes Inc., Vineland, NJ). In a second step, 3YTZP + SWNTs powder solutions were mixed in aqueous solution and subjected to ultrasonic agitation for $15 \mathrm{~min}$ by means of the ultrasonic bath/probe, followed by drying on a hot plate while being stirred. Finally, composite powders were homogenized in agate mortar. 
SPS (Model 515S, SPS Dr Sinter Inc., Kanagawa, Japan) was used to sinter the materials in a $15-\mathrm{mm}$ diameter cylindrical graphite die/punch setup in vacuum atmosphere, using the following sintering conditions: sintering temperature of $1250^{\circ} \mathrm{C}$ for $5 \mathrm{~min}$, applied uniaxial pressure of $75 \mathrm{MPa}$, and heating and cooling rates of 300 and $50^{\circ} \mathrm{C} / \mathrm{min}$, respectively. Bulk densities were measured using Archimedes' method, with distilled water as immersion medium. Theoretical density values for composites were calculated by the rule of mixtures assuming density values of $6.10 \mathrm{~g} \cdot \mathrm{cm}^{-3}$ for 3YTZP and $1.80 \mathrm{~g} \cdot \mathrm{cm}^{-3}$ for SWNTs.

\subsection{Microstructural and electrical characterization}

Phase identification was carried out using X-ray diffraction (XRD, model D8 Advance A25, Bruker Co., Massachusetts,USA). Structural integrity of SWNTs in the composites after SPS sintering was assessed by Raman spectroscopy on fracture surfaces using a dispersive microscope (Horiba Jobin Yvon LabRam HR800, Kyoto, Japan) equipped with a $20 \mathrm{~mW}$ He-Ne green laser $(\lambda=532.14 \mathrm{~nm})$. The microscope used a 100x objective and a confocal pinhole of $100 \mu \mathrm{m}$.

Microstructural studies of composite fracture surfaces were performed by highresolution scanning electron microscopy HRSEM (HITACHI S5200) to analyze the distribution of SWNTs in the zirconia matrix. Polished surfaces to characterize the ceramic 3 YTZP grains morphology by HRSEM were thermally etched at $1200{ }^{\circ} \mathrm{C}$ for $20 \mathrm{~min}$ in air to reveal grain boundaries. The morphological characterization was made measuring 200 grains to obtain the equivalent planar diameter as size parameter, $d$ $=2(\operatorname{area} / \pi)^{1 / 2}$, and the shape factor, $f=(4 \pi \cdot$ area $) /(\text { perimeter })^{2}$. Standard deviation of distributions was also evaluated. ImageJ software was used for morphological analysis. 
Electrical characterization was carried out by Impedance Spectroscopy using an Agilent 4294A analyzer in the frequency range from 100 to $2 \times 10^{6} \mathrm{~Hz}$, at temperatures from 25 to $450{ }^{\circ} \mathrm{C}$. Measurements were carried out in argon atmosphere to avoid oxidation of the samples and subsequent degradation of the SWNTs during the process. Colloidal silver paste was applied on both sides of the samples and electrodes were fired at $600{ }^{\circ} \mathrm{C}$ for $30 \mathrm{~min}$ under argon flow. Equivalent circuit approach was adopted for the data analysis with fitted curve using Z-view software and equivalent circuit model.

\section{Results and discussion}

\subsection{Microstructural characterization}

Microstructural data for ceramic grains in the composites prepared using the ultrasonic probe, together with density values, are shown in Table 1. Relative densities higher than $98 \%$ were obtained in the three composites, so an almost full densification was obtained with the selected sintering parameters. Similar mean grain size and shape factor were measured in the composites with different SWNT vol\% and no significant differences were observed compared to the composites prepared using the ultrasonic bath. $^{17}$

Raman spectra of the composites (shown in supplementary information) revealed the presence of SWNT characteristic bands, in particular the low-frequency radial breathing mode (RBM) and the $\mathrm{G}$ band, confirming absence of significant damage to the SWNTs during processing and sintering. D band, associated with crystalline defects, was also observed. $\mathrm{I}_{\mathrm{D}} / \mathrm{I}_{\mathrm{G}}$ ratio calculations for the composites prepared with the high energy ultrasonic probe (3.0, 6.3 and $7.9 \%$ for $0.5,1$ and 1.5 vol\% SWNT, respectively) gave values similar to those published ${ }^{17}$ for the composites 
prepared with the ultrasonic bath $(6.6,6.5$ and $7.2 \%$ for $0.5,1$ and $1.5 \mathrm{vol} \% \mathrm{SWNT}$, respectively), pointing to a similar amount of crystalline defects in the nanotubes.

XRD patterns of the sintered composites are shown in Fig. 1. In all the samples, it is clear the presence of the tetragonal phase (JCPDS 01-078-1808) as the main one, with a contribution of the monoclinic phase (JCPDS 01-081-1314). A higher intensity of the main peak corresponding to the monoclinic phase (at $28.2^{\circ} 2 \theta$ ) is observed in the composites prepared using ultrasonic probe (Fig. 1b), pointing to a higher contribution of this phase. Also, an increase of this peak is clearly observed when increasing the SWNT content in both sets of composites. Recently, our group reported ${ }^{19}$ an increase of the monoclinic phase contribution in high SWNT content 3YTZP composites when increasing the SWNT content from 2.5 to $10 \mathrm{vol} \%$, which we related to a tetragonal to monoclinic transformation promoted by the presence of carbon nanotubes in the microstructure. Such transformation was also evidenced by Bocanegra-Bernal et al. ${ }^{23}$ for zirconia toughened alumina (ZTA)/0.01 wt $\%$ MWNT composites pressureless sintered in air using graphite as bed powder. The SWNTs surrounding the zirconia grains generate a reducing atmosphere during sintering, which promotes the partial destabilization of the tetragonal phase and thus, the presence of the monoclinic one. For high SWNT content, we showed that the higher the SWNT vol\%, the higher the monoclinic phase contribution.

In this study, the higher contribution of the monoclinic phase obtained for the same SWNT contents in the composites prepared using the ultrasonic probe points to a better SWNT distribution in the ceramic matrix in these composites. We have shown that processing of these composites by means of the ultrasonic bath is not successful in completely avoiding SWNT agglomeration. ${ }^{17} 36 \%, 26 \%$ and $27 \%$ of the nominal carbon nanotubes were contained in agglomerates for composites with $0.5,1$ and 1.5 
nominal SWNT vol\%, respectively, and the real contents of nanotubes at the grain boundaries were estimated to be $0.32,0.74$ and $1.1 \mathrm{SWNT}$ vol\%. When using the ultrasonic probe a lower amount of agglomerates is expected ${ }^{21}$ and a higher content of nanotubes surrounding the zirconia grains will promote the destabilization of the tetragonal phase, giving place to a higher contribution of the monoclinic one.

HRSEM images (Fig. 2) show the typical appearance of the fracture surfaces of the composites prepared by using the ultrasonic probe, illustrating the adequate dispersion of SWNT bundles surrounding zirconia grains. The fracture mode is mainly intergranular and debonded CNTs from the matrix can be observed on the fracture surfaces. CNT agglomerates were scarcely found in these high-resolution observations.

\subsection{Electrical characterization}

Figure 3 shows the impedance plots acquired from room temperature up to 180 ${ }^{\circ} \mathrm{C}$ in the composites with 1 and 1.5 vol\% SWNT prepared by using the ultrasonic probe. A single impedance arc can be observed for both composites until $180^{\circ} \mathrm{C}$, when a second arc appears at lower frequency. For this set of samples, at room temperature only the composite with $0.5 \mathrm{vol} \% \mathrm{SWNT}$ was found to be electrically isolating with a very high room temperature resistivity. Room temperature conductivities of $1.7 \times 10^{-4}$ and $2.8 \times 10^{-4} \mathrm{~S} \cdot \mathrm{cm}^{-1}$ were measured in the composites with 1 and $1.5 \mathrm{vol} \% \mathrm{SWNT}$, respectively. It can be concluded that the percolation threshold of the carbon nanotubes in the ceramic matrix is between 0.5 and $1 \mathrm{vol} \%$, which is lower than the published result for the composites prepared with the ultrasonic bath (threshold between 1 and 1.5 vol $\% \mathrm{SWNT}$ ). The value obtained in this study is in good agreement with the values of $\sim 0.6$ vol\% published by Rul et al. ${ }^{24}$ and Poorteman et al., ${ }^{12}$ or $\sim 0.8$ vol $\%$ published by Ahmad et al. ${ }^{9}$ for $\mathrm{Al}_{2} \mathrm{O}_{3} / \mathrm{MWNT}$ composites. However, it is lower that the published 
ones for zirconia/CNT composites, $\sim 1.7$ vol $\%$ or 5.5 vol $\%$ reported by Shin et al. ${ }^{13}$ and Shi et al., ${ }^{10,11}$ respectively. This is consequence of the low presence of agglomerates in our composites, leading to a higher amount of SWNT distributed in the ceramic grain boundaries.

Remarkably higher values of room temperature conductivity have been achieved in the composites when using the ultrasonic probe, in comparison with the conductivity of the composite with $1.5 \mathrm{vol} \%$ SWNT prepared using the ultrasonic bath $\left(\sigma=6 \times 10^{-6}\right.$ $\left.\mathrm{S} \cdot \mathrm{cm}^{-1}\right) .{ }^{17}$ Thus, it is clear that the decrease of the percolation limit and the increase of the room temperature conductivity are consequences of the excellent dispersion of the carbon nanotubes throughout the ceramic matrix achieved by introducing the use of the ultrasonic probe in the processing routine.

When impedance spectroscopy measurements are carried out in carbon nanotube conducting networks, and considering that the network is formed by carbon nanotube bundles and junctions between these bundles, the characteristic length scale of the network can be associated with the distance between junctions in the SWNT network. ${ }^{25}$ The transition from long-range transport (frequencies are lower than the correlation frequency and charge carriers move over long distances) to localized carrier confinement (higher frequencies, charge carriers move only within the bundles) is characterized by a falloff in the real impedance with increasing frequency, and a maximum in the imaginary impedance (Fig 4). It can be observed the increase of the falloff frequency from 25 to $55 \mathrm{kHz}$, and the decrease of real impedance from 5.8 to 3.5 $\mathrm{k} \Omega \cdot \mathrm{cm}$, when increasing the SWNT vol\% from 1 to 1.5 . This result is similar to the reported one by Garrett et al..$^{25}$ for thin films of SWNT networks prepared with different SWNT loadings. 
In this context, when modelling the impedance properties of a ceramic composite with a SWNT percolated network, an equivalent circuit model consisting of two R-C elements in series describes suitably the conduction through SWNT junctions and bundles. ${ }^{17,25,26}$ It has been established by different authors that the resistance across junctions is higher than the resistance through the carbon nanotube bundles themselves. ${ }^{27-29}$ Thus, the lower resistance element will be assigned to the SWNT bundles and the higher resistance one will be assigned to the junctions between bundles.

Table 2 displays the fitting parameters (resistivity and capacitance) of the single impedance arc at room temperature obtained for the composites with 1 and $1.5 \mathrm{vol} \%$ SWNT prepared by using the ultrasonic probe. Parameters reported for the composite with 1.5 vol\% SWNT prepared by using the ultrasonic bath were included in order to establish a comparison. A remarkable decrease of both bundles and junctions resistivities is observed in the composites when replacing the ultrasonic bath by the probe in the processing routine. In our previous study, ${ }^{17}$ the observed high junction resistivity was related to the existence of junctions involving large diameter bundles or SWNT agglomerates, on the other hand, previous authors had shown that the junction resistance is strongly dependent on the size of the interconnecting bundles, with the smallest values associated with individual tubes. ${ }^{28}$ Thus, the processing efforts devoted in this study to minimize the presence of SWNT agglomerates in the composites, have clearly leaded to reduced bundle diameter, resulting also in a reduced junction resistivity and an increased total conductivity.

In order to analyse the evolution of SWNT bundles and junctions resistivities with temperature we have modelled the single impedance arc obtained for the composites from room temperature up to $180^{\circ} \mathrm{C}$, using the previously described equivalent circuit. Results of the modeling for both composites in this temperature range 
are shown in figure 5(a). Evolution of resistivities for the composite with $1.5 \mathrm{vol} \%$ SWNT prepared using the ultrasonic bath ${ }^{17}$ has been reprinted in figure 5(b) for comparison. For the three composites it is observed that, whereas the SWNT bundle resistivity is almost constant with temperature, a decrease of junctions' resistivity takes place. Nevertheless, this decrease is significantly more remarkable for the composite prepared using the ultrasonic bath $\left(65 \%\right.$ decrease from room temperature to $180{ }^{\circ} \mathrm{C}$ versus $25 \%$ for the composites prepared using the ultrasonic probe). The decrease of junctions' resistivity with temperature can be successfully described in terms of the fluctuation-induced electron tunneling across nanotube-nanotube junctions. ${ }^{17,30}$ Briefly, the small insulating barriers separating the conducting regions in the system are very susceptible to charge fluctuations, resulting in electric field fluctuations across the tunneling junctions. These fluctuations increase with temperature, giving place to a decrease of junctions' resistivity. It is clear that this effect is more remarkable in the case of the composite with large diameter bundles or SWNT agglomerates than in the case of the composite with reduced bundle diameter achieved by using the ultrasonic probe, resulting in the latter case in a smooth decrease of junctions' resistivity with temperature.

Figure 6 shows the impedance plots, in the complex plane, acquired from 200 to $320^{\circ} \mathrm{C}$ in the composites with 1 and $1.5 \mathrm{vol} \% \mathrm{SWNT}$ prepared by using the ultrasonic probe. Two impedance arcs are observed, with a different evolution with increasing temperature. Whereas the arc at higher frequency decreases monotonously with temperature, the arc at lower frequency increases up to $270-280{ }^{\circ} \mathrm{C}$ and decreases for higher temperatures. This unusual behaviour is also observed in the $Z$ " versus f spectra where two peaks are observed, the one at higher frequency decreasing monotonously with temperature, and the one at lower frequency increasing and decreasing. 
This behaviour is quite different from the typically described for an ionic conductor, with two arcs in the impedance plot, or two peaks in the Z" spectrum, that decrease monotonically with temperature, the higher frequency one corresponding to conductivity through the ceramic bulk and the lower frequency one corresponding to conductivity through the grain boundaries.

Figure 7 shows the dependence of $\mathrm{f}_{\mathrm{Max} 1}$ and $\mathrm{f}_{\mathrm{Max} 2}$ versus temperature, where $f_{\text {Max } 1}$ and $f_{\text {Max } 2}$ are the frequencies of the maxima of the two peaks observed in the $Z$ " versus $f$ spectra, for the three composites with a percolating network. $f_{\text {Max } 1}$ corresponds to the peak at higher frequency and is represented with solid symbols, and $\mathrm{f}_{\mathrm{Max} 2}$ corresponds to the peak at lower frequency and is represented with open symbols. These frequencies will allow identification of the different contributions to conductivity contained in the two different impedance arcs/peaks for the 3YTZP/SWNT composites. Data corresponding to a monolithic 3YTZP ceramic, sintered with the same conditions, have been included in order to establish a comparison. In the case of $f_{\text {Max } 1}$, it can be observed that for the low temperature range the trend for the composites is not similar to the typical one of an ionic conductor, so it can be concluded that the higher frequency peak in the composites describes only the electronic conductivity through the SWNT network. For higher temperatures, the frequency in the composites tends to the one corresponding to the ionic contribution to conductivity through the ceramic bulk. Thus, in the high temperature range a mixed ionic-electronic conductivity is described by the higher frequency peak. Regarding $\mathrm{f}_{\mathrm{Max} 2}$, it can be observed that this frequency in the composites is close to the one corresponding to the monolithic ceramic in the whole range of temperatures. Thus, it can be concluded that the lower frequency peak in the composites describes the ionic conductivity through the grain boundaries in the 3 YTZP ceramic. However, it has to be considered that a great fraction of the grain boundaries is 
covered by SWNT in the composites. This fact was pointed as the main cause of the unusual behaviour of the low frequency arc with temperature (increasing and decreasing with an inflection point at $350^{\circ} \mathrm{C}$ ) in the composite with 1.5 vol\% SWNT prepared using the ultrasonic bath. ${ }^{17}$ The SWNTs distributed at the ceramic grain boundaries reduce the ionic conductivity increasing the grain boundary resistivity until a certain temperature, when this effect is overcome and the grain boundary conductivity increases with temperature. It is interesting to note that this temperature is remarkably lower in the composites prepared using the ultrasonic probe in comparison with the reported value for the composite with 1.5 vol\% SWNT prepared using the ultrasonic bath (270$280{ }^{\circ} \mathrm{C}$ and $350{ }^{\circ} \mathrm{C}$, respectively). This result should be consequence of the reduced bundle diameter achieved in this study, as a lower temperature is needed by the ionic conduction to overcome the blocking effect of the SWNT distributed at the ceramic grain boundaries.

Arrhenius plots for total conductivity up to $340^{\circ} \mathrm{C}$ in the composites with a percolated network are shown in figure 8. Data corresponding to a monolithic 3YTZP ceramic, sintered with the same conditions, have been included in order to establish a comparison. The slope of these diagrams was used to calculate the activation energy of the conducting species (Table 3). Significant differences are observed between the activation energies in the composites and in the monolithic ceramic. For the 3 YTZP ceramic a value close to $0.8 \mathrm{eV}$ was obtained. This value is consistent with the activation energy for the oxygen vacancies ionic conductivity in zirconia-based ceramic oxides. ${ }^{31}$ However, significantly lower activation energies were obtained for the composites. On the other hand, remarkable differences are also observed between the composites prepared with the two different processing routines. Whereas for the composite prepared using the ultrasonic bath a single activation energy is observed in 
the whole temperature range $(65 \mathrm{meV})$ for the composites prepared using the ultrasonic probe two different activation energies are observed $(\sim 20 \mathrm{meV}$ from room temperature to $260{ }^{\circ} \mathrm{C}$ and $\sim 0.4 \mathrm{eV}$ from $260^{\circ} \mathrm{C}$ to $340{ }^{\circ} \mathrm{C}$ ). From these activation energy values, the remarkable effect of SWNTs on the electric behaviour of the composites is evidenced. In the low temperature range, the low activation energy is pointing to a charge transport dominated by the SWNT contribution. This activation energy is similar to the reported value for $8 \mathrm{~mol} \% \mathrm{Y}_{2} \mathrm{O}_{3}-\mathrm{ZrO}_{2} / \mathrm{SWNT}$ composites $(30 \mathrm{meV}) .{ }^{14}$ For higher temperatures, the increase of the activation energy reveals the contribution of the ionic conduction giving place to a mixed ionic-electronic conduction.

\section{Conclusions}

Highly dense 3 mol\% yttria doped zirconia matrix composites containing $0.5,1$ and 1.5 vol\% SWNT, with analogous ceramic grain size and mainly tetragonal phase with a minor contribution of monoclinic phase were prepared by two different colloidal powder processing routines and SPS. An enhanced SWNT distribution throughout the 3YTZP ceramic matrix was obtained in the composites prepared by aqueous colloidal powder processing using an ultrasonic probe, as revealed by the higher contribution of the monoclinic phase in these composites, consequence of the transformation of the tetragonal phase to the monoclinic one promoted by the presence of SWNTs at the grain boundaries. A decrease of the percolation threshold of the carbon nanotubes in the 3YTZP matrix and an increase of the room temperature conductivity were also consequences of this optimal SWNT distribution.

Modelling of the impedance properties allowed separation of the individual SWNT bundles contribution to resistance from the resistance due to junctions between 
bundles in the composites with a percolating network. The remarkable decrease of both SWNT bundles and junctions' resistivity together with the smooth decrease of junctions' resistivity with temperature observed in the composites when replacing the ultrasonic bath by the probe in the powder processing routine pointed to a minimized presence of SWNT agglomerates together with a reduced SWNT bundle diameter in these composites.

Analysis of the frequencies of the maxima of the two peaks observed in the Z" vs f spectra allowed identification of the different contributions to conductivity, pointing to electronic conduction through the SWNTs at low temperatures and a mixed electronic-ionic conductivity through the ceramic bulk in the high temperature range. A blocking effect to the ionic conductivity through the grain boundary was evidenced and related to the presence of SWNTs at the grain boundaries. This effect was overcome at a lower temperature in the composites prepared by using the ultrasonic probe, as a result of the reduced SWNT bundle diameter achieved in these composites.

\section{Acknowledgements}

The financial support for this work has been obtained from the Spanish Ministry of Science and Innovation (MAT2012-34217) and from Junta de Andalucía (P12-FQM1079). XRD and microscopy studies have been performed in facilities belonging to the CITIUS (Universidad de Sevilla). 


\section{Figure captions}

Figure 1: X-ray diffraction patterns of the composites prepared by means of (a) ultrasonic bath, and (b) ultrasonic probe.

Figure 2: HRSEM micrographs of fracture surface of the composites prepared by means of the ultrasonic probe with (a) $0.5 \mathrm{vol} \%$, (b) $1 \mathrm{vol} \%$, and (c) $1.5 \mathrm{vol} \% \mathrm{SWNT}$.

Figure 3: Impedance plots acquired from room temperature to $180^{\circ} \mathrm{C}$ in the composites prepared by means of the ultrasonic probe with (a) 1 vol $\%$, and (b) 1.5 vol\% SWNT.

Figure 4: Frequency dependence of the real and imaginary impedance at room temperature for the composites with 1 and $1.5 \mathrm{vol} \% \mathrm{SWNT}$, prepared by means of the ultrasonic probe.

Figure 5: SWNT bundles and junctions resistivities from room temperature to $180{ }^{\circ} \mathrm{C}$ for (a) the composites with 1 and $1.5 \mathrm{vol} \% \mathrm{SWNT}$, prepared by means of the ultrasonic probe, and (b) the composite with $1.5 \mathrm{vol} \% \mathrm{SWNT}$, prepared by means of the ultrasonic bath (from reference 17).

Figure 6: Impedance plots acquired at temperatures higher than $200{ }^{\circ} \mathrm{C}$ in the composites prepared by means of the ultrasonic probe with (a) 1 and (b) $1.5 \mathrm{vol} \%$ SWNT. 
Figure 7: Representation of the dependence of frequency of the maximum of the peaks in the Z" versus f spectra, with temperature for the composites with 1 and $1.5 \mathrm{vol} \%$ SWNT, prepared by means of the ultrasonic probe, and the composite with $1.5 \mathrm{vol} \%$ SWNT, prepared by means of the ultrasonic bath.

Figure 8: Arrhenius plots of total resistivity for the composites with 1 and 1.5 vol\% SWNT, prepared by means of the ultrasonic probe, and the composite with $1.5 \mathrm{vol} \%$ SWNT, prepared by means of the ultrasonic bath. 
Table 1: Relative density $\left(\rho_{r}\right)$ and morphological parameters (average grain size $d$, shape factor $F$, grain size and shape factor standard deviations, $\sigma_{<d>}$ and $\sigma_{<F>}$ ) for ceramic grains in the composites prepared using the ultrasonic probe.

\begin{tabular}{|c|c|c|c|c|c|}
\hline SWNT vol\% & $\begin{array}{c}\boldsymbol{\rho}_{\mathbf{r}} \pm \mathbf{0 . 5} \\
\mathbf{( \% )}\end{array}$ & $\begin{array}{c}\mathbf{d} \\
(\mathbf{n m})\end{array}$ & $\begin{array}{c}\boldsymbol{\sigma}_{<\mathbf{d}>} \\
(\mathbf{n m})\end{array}$ & $\mathbf{F}$ & $\boldsymbol{\sigma}_{<\mathbf{F}>}$ \\
\hline 0.5 & 98.5 & 240 & 80 & 0.76 & 0.05 \\
\hline 1.0 & 98.9 & 240 & 80 & 0.74 & 0.08 \\
\hline 1.5 & 99.5 & 220 & 80 & 0.75 & 0.06 \\
\hline
\end{tabular}


Table 2: Equivalent circuit values for the fitting of the impedance properties at room temperature of the composites with a percolated network. $\rho_{J}, C_{J}$ and $\rho_{B}, C_{B}$ describe the electrical properties of the junctions and nanotubes bundles, respectively.

\begin{tabular}{|c|c|c|c|c|c|}
\hline SWNT vol\% & & $\boldsymbol{\rho}_{\mathbf{J}}(\mathbf{k} \boldsymbol{\Omega} \cdot \mathbf{c m})$ & $\mathbf{C}_{\mathbf{J}}(\mathbf{p F})$ & $\boldsymbol{\rho}_{\mathbf{B}}(\mathbf{k} \mathbf{\Omega} \cdot \mathbf{c m})$ & $\mathbf{C}_{\mathbf{B}}(\mathbf{p F})$ \\
\hline $1.5^{*}$ & bath & $160 \pm 13$ & $272 \pm 3$ & $10.4 \pm 0.9$ & $137 \pm 3$ \\
\hline 1.5 & probe & $3.4 \pm 0.6$ & $481 \pm 1$ & $0.21 \pm 0.04$ & $39 \pm 1$ \\
\hline 1.0 & probe & $5.6 \pm 0.4$ & $1585 \pm 3$ & $0.28 \pm 0.02$ & $123 \pm 2$ \\
\hline
\end{tabular}

*From reference 17 
Table 3: Activation energy of the conducting species for the composites with a percolated network.

\begin{tabular}{|c|c|c|c|}
\hline \multirow{2}{*}{ SWNT vol\% } & \multirow{2}{*}{} & \multicolumn{2}{|c|}{$\mathbf{E}_{\text {act }}(\mathbf{e V})$} \\
\cline { 2 - 4 } & & RT - 260 & \\
\hline $0.0^{*}$ & --- & $\mathbf{2 6 0}^{\circ} \mathbf{C}-\mathbf{3 4 0}^{\circ} \mathbf{C}$ \\
\hline $1.5^{*}$ & bath & \multicolumn{2}{|c|}{$0.86 \pm 0.02$} \\
\hline 1.5 & probe & $0.018 \pm 0.002$ & $0.34 \pm 0.03$ \\
\hline 1.0 & probe & $0.025 \pm 0.003$ & $0.46 \pm 0.03$ \\
\hline
\end{tabular}

*From reference 17 

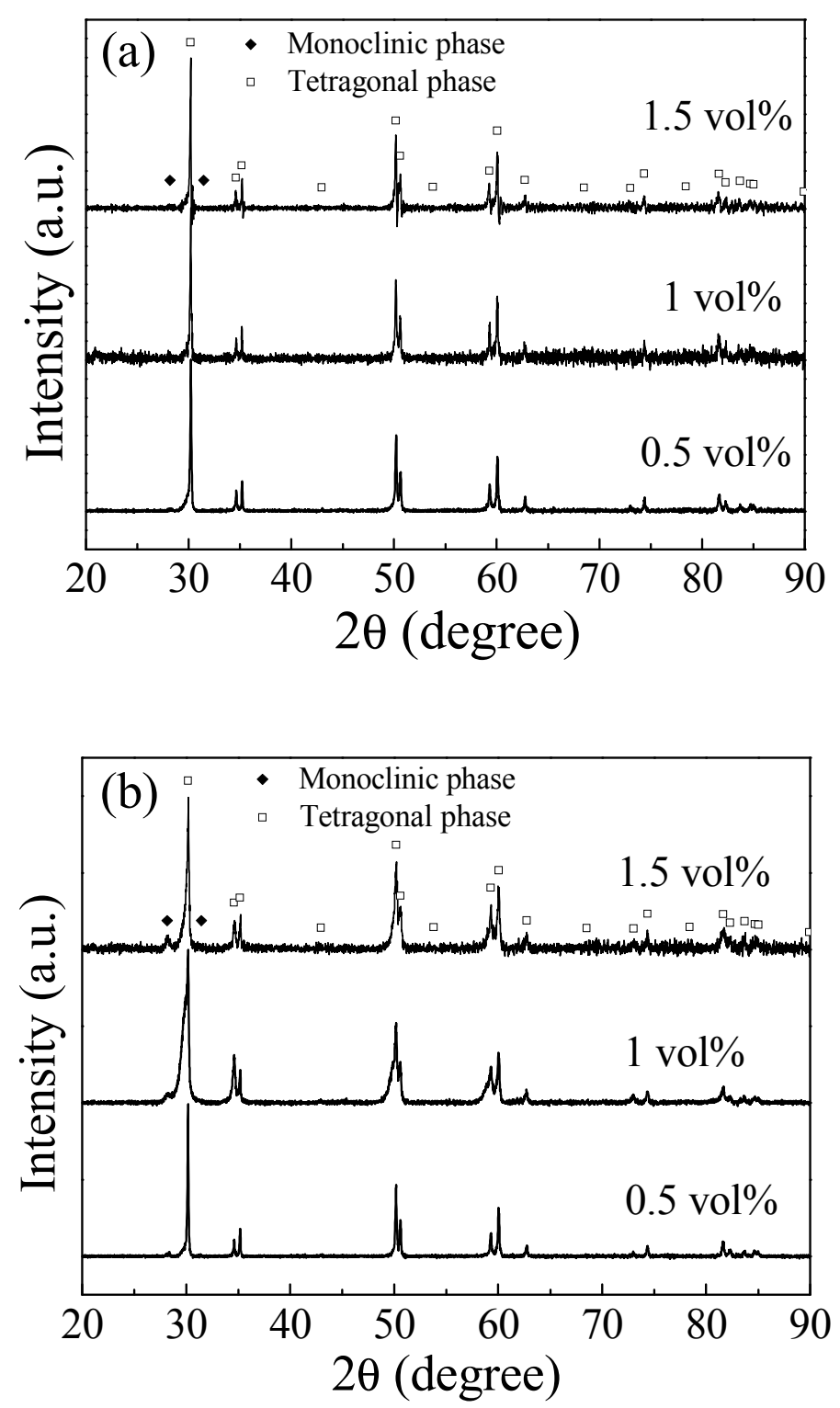

Figure 1 

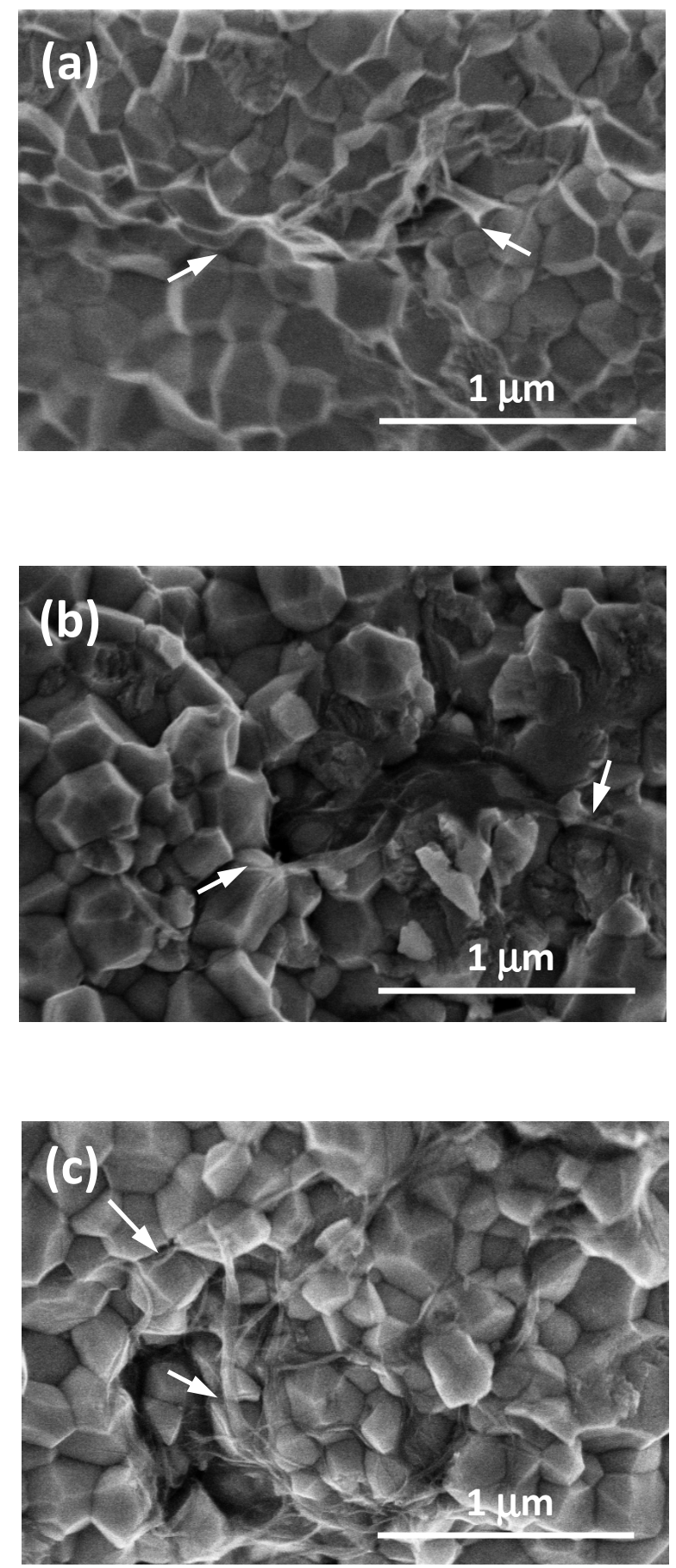

Figure 2 

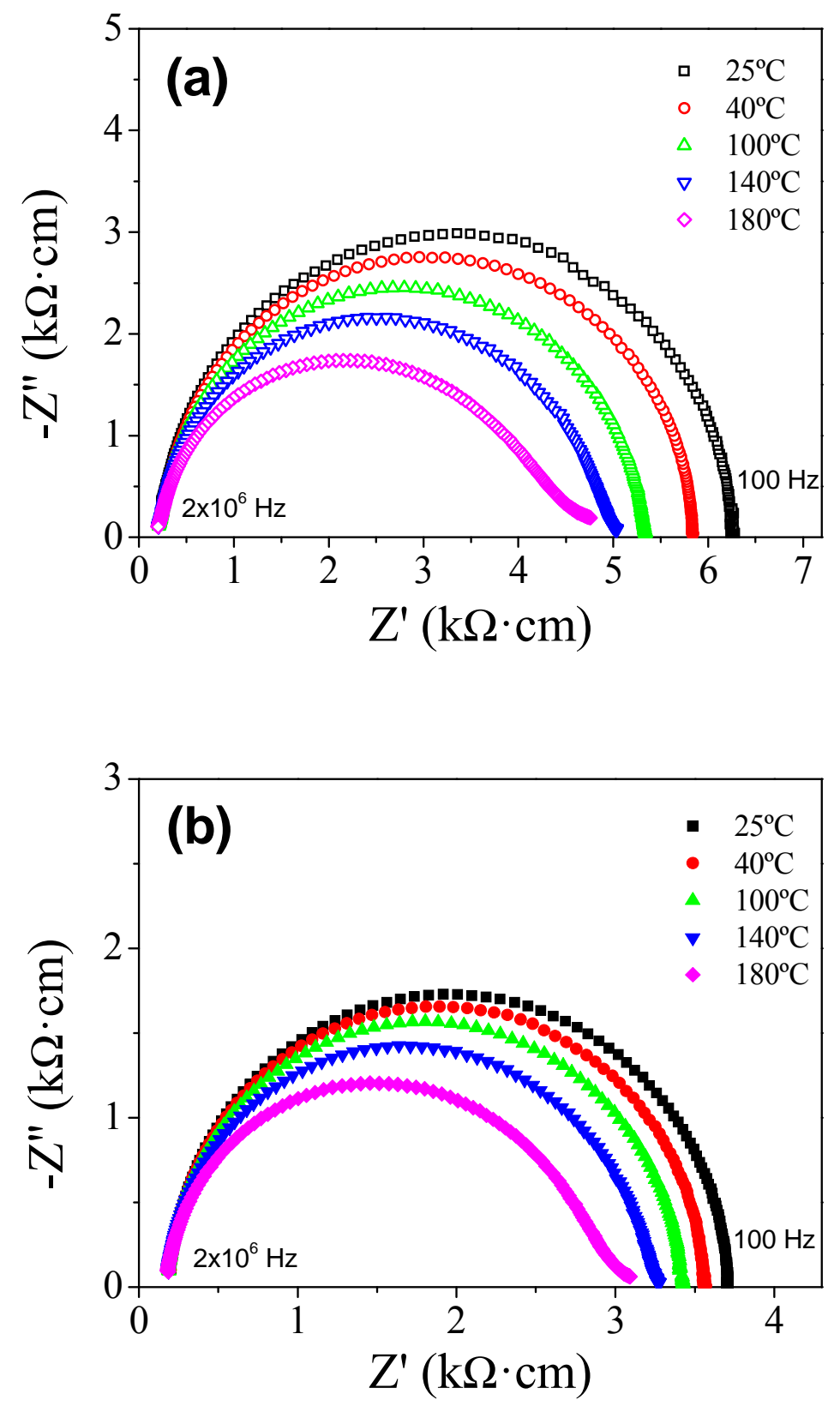

Figure 3 


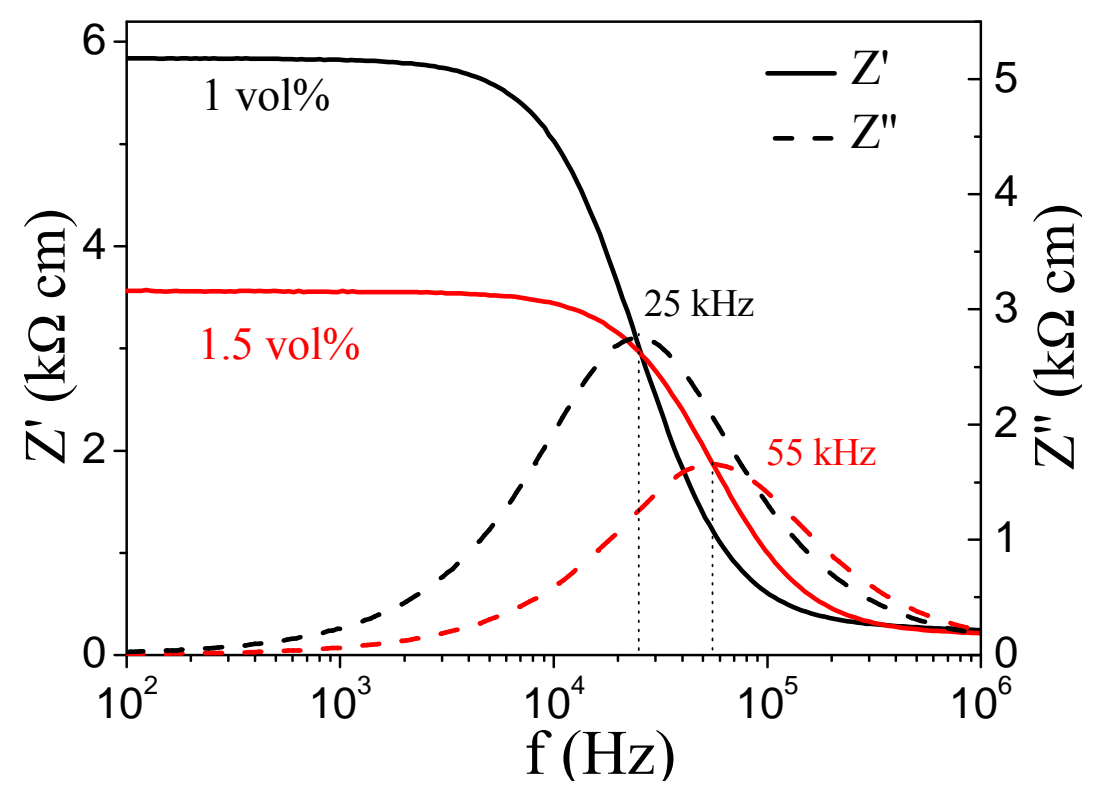

Figure 4 

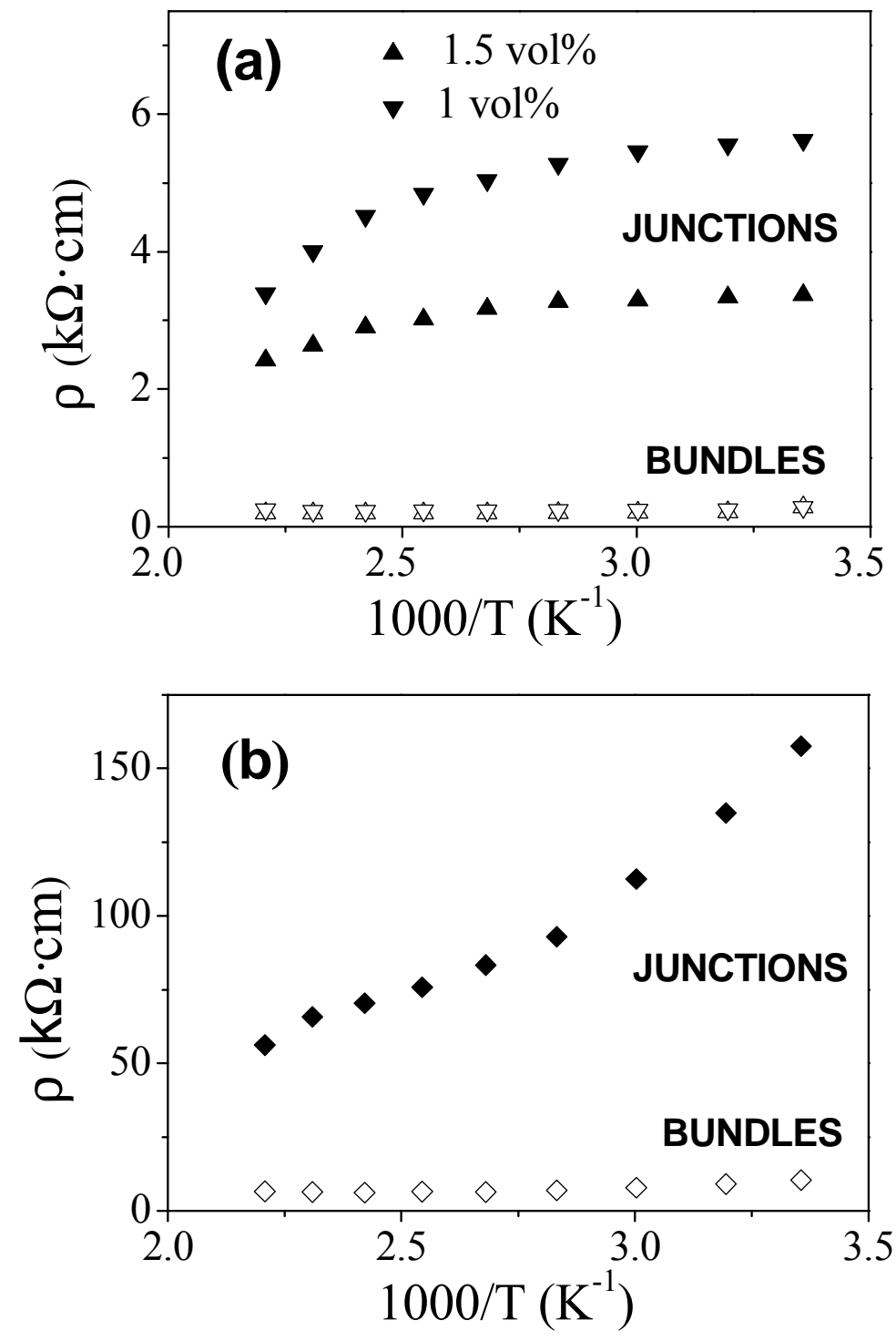

Figure 5 

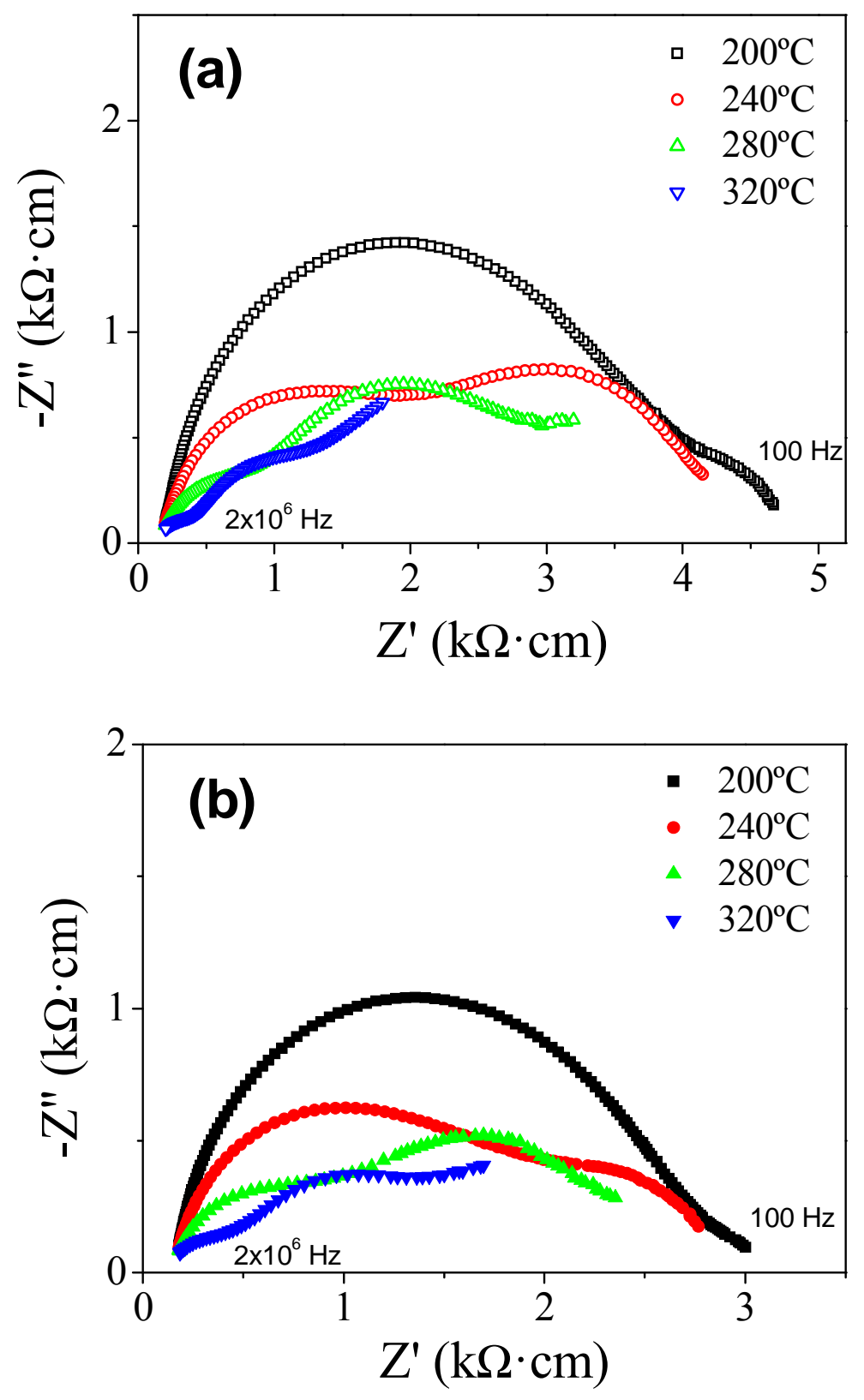

Figure 6 


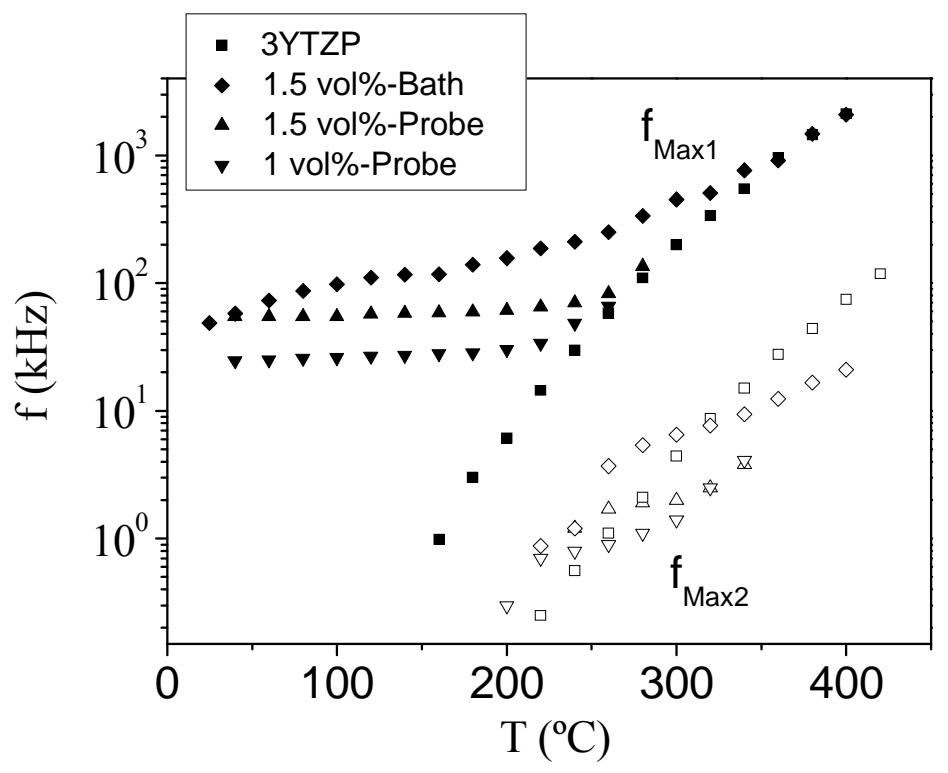

Figure 7 


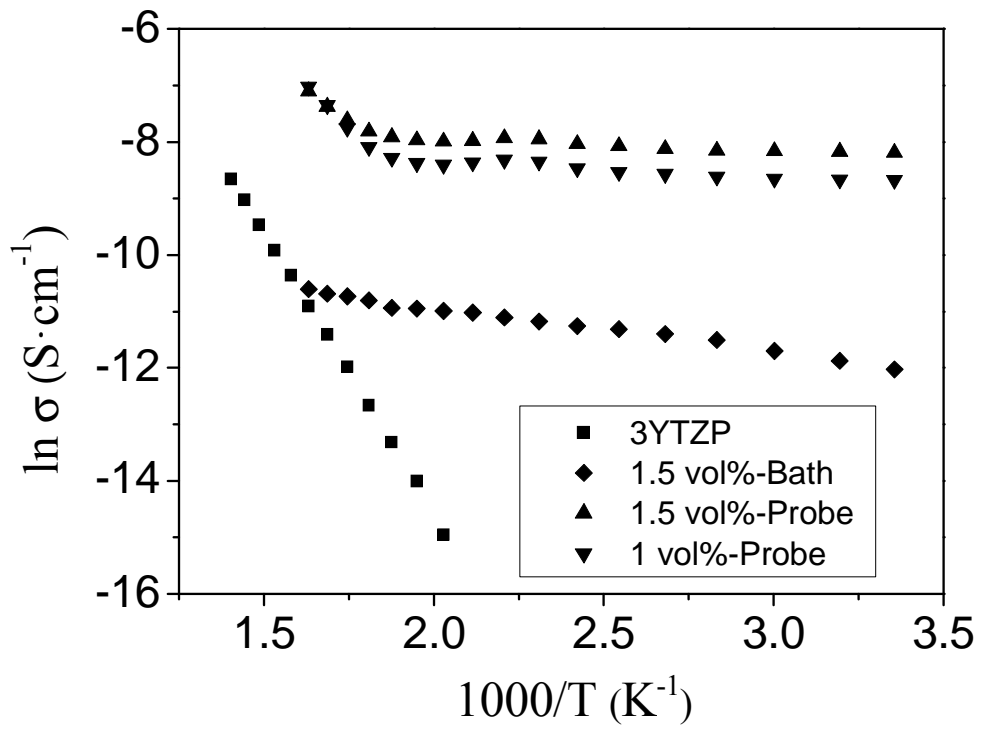

Figure 8 


\section{Supplementary Information}

Raman spectra measured in the composites prepared by means of the ultrasonic probe, including the RBM frequency range and the D and G-Bands frequency range.

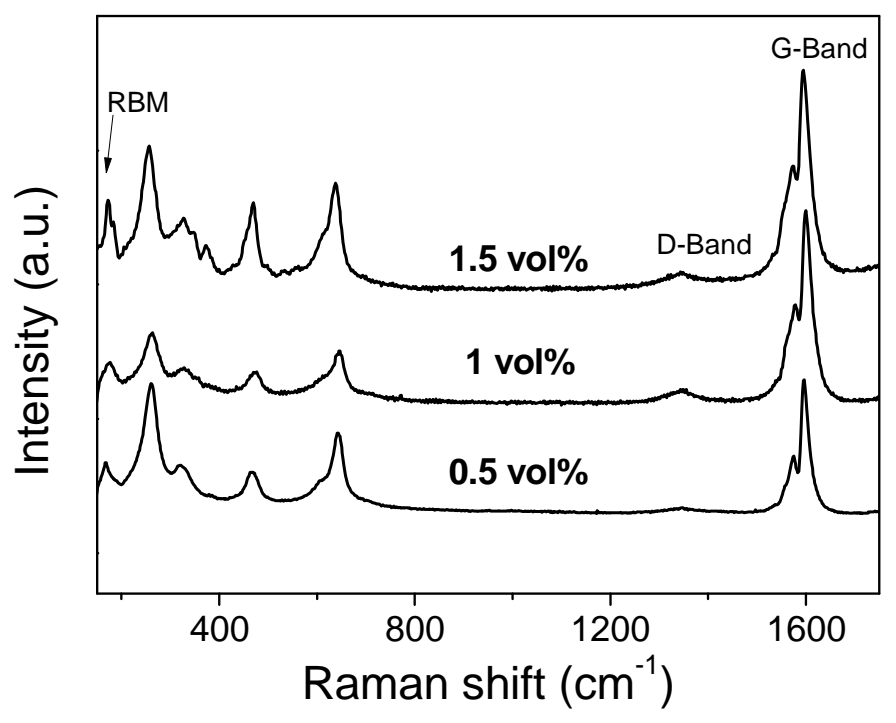




\section{References}

${ }^{1}$ I. Ahmad, B. Yazdani, Y. Zhu, Recent advances on carbon nanotubes and graphene reinforced ceramics nanocomposites, Nanomaterials 5 (2015) 90-114.

${ }^{2}$ M. Endo, M.S. Strano, P.M. Ajayan, Potential applications of carbon nanotubes, Top Appl. Phys. 111 (2008) 13-61.

${ }^{3}$ R.H. Baughman, A.A. Zakhidov, W.A. de Heer, Carbon nanotubes - the route toward applications, Science 297 (2002) 787-792.

${ }^{4}$ N.P. Padture, Multifunctional composites of ceramics and single-walled carbon nanotubes, Adv. Mater. 21 (2009) 1767-1770.

${ }^{5}$ F. Inam, H. Yan, D.D. Jayaseelan, T. Peijs, M. J. Reece, Electrically conductive alumina-carbon nanocomposites prepared by Spark Plasma Sintering, J. Eur. Ceram. Soc. 30 (2010) 153-157.

${ }^{6}$ E.L. Corral, H. Wang, J. Garay, Z. Munir, E.V. Barrera, Effect of single-walled carbon nanotubes on thermal and electrical properties of silicon nitride processed using spark plasma sintering, J. Eur. Ceram. Soc. 31 (2011) 391-400.

${ }^{7}$ A. Kasperski, A. Weibel, C. Estournès, Ch. Laurent, A. Peigney, Preparationmicrostructure-property relationships in double-walled carbon nanotubes/alumina composites, Carbon 53 (2013) 62-72.

${ }^{8}$ A. Kovalcíková, Cs. Balázsi, J. Dusza, O. Tapasztó, Mechanical properties and electrical conductivity in a carbon nanotube reinforced silicon nitride composite, Ceramics International 38 (2012) 527-533. 
${ }^{9}$ K. Ahmad, W. Pan, S.L. Shi, Electrical conductivity and dielectric properties of multiwalled carbon nanotube and alumina composites, Appl. Phys. Lett. 89 (2006) 133122.

${ }^{10}$ S.L. Shi, J. Liang, Effect of multiwall carbon nanotubes on electrical and dielectric properties of yttria-stabilized zirconia ceramic, J. Am. Ceram. Soc. 89 (2006) 35333535 .

${ }^{11}$ S.L. Shi, J. Liang, Electronic transport properties of multiwall carbon nanotubes/yttria stabilized zirconia composites, J. Appl. Phys. 101 (2007) 023708.

${ }^{12}$ M. Poorteman, M. Traianidis, G. Bister, F. Cambier, Colloidal processing, hot pressing and characterisation of electroconductive MWCNT-alumina composites with compositions near the percolation threshold, J. Eur. Ceram. Soc. 29 (2009) 669-675.

${ }^{13}$ J.H. Shin, S.H. Hong, Microstructure and mechanical properties of single wall carbon nanotube reinforced yttria stabilized zirconia ceramics, Mater. Sci. Eng. A 556 (2012) $382-387$.

${ }^{14}$ F.C. Fonseca, R. Muccillo, D.Z. de Florio, L.O. Ladeira, A.S. Ferlauto, Mixed ionicelectronic conductivity in yttria-stabilized zirconia/carbon nanotube composites, Appl. Phys. Lett. 91 (2007) 243107.

${ }^{15}$ K. Ahmad, W. Pan, Dramatic effect of multiwalled carbon nanotubes on the electrical properties of alumina based ceramic nanocomposites, Compos. Sci. Technol. 69 (2009) $1016-1021$. 
${ }^{16}$ J. González-Julián, Y. Iglesias, A.C. Caballero, M. Belmonte, L. Garzón, C. Ocal, et al. Multi-scale electrical response of silicon nitride/multi-walled carbon nanotubes composites, Compos. Sci. Technol. 71 (2011) 60-66.

${ }^{17}$ R. Poyato, J. Macías-Delgado, A. García-Valenzuela, A. Gallardo-López, A. Morales-Rodríguez, A. Muñoz, A. Domínguez-Rodríguez, Mechanical and electrical properties of low SWNT content 3YTZP composites, J. Eur. Ceram. Soc. 35 (2015) $2351-2359$.

${ }^{18}$ R. Poyato, A.L. Vasiliev, N.P. Padture, H. Tanaka, T. Nishimura, Aqueous colloidal processing of single-wall carbon nanotubes and their composites with ceramics, Nanotechnology 17 (2006) 1770-1777.

${ }^{19}$ R. Poyato, A. Gallardo-López, F. Gutiérrez-Mora, A. Morales-Rodríguez, A. Muñoz, A. Domínguez-Rodríguez, Effect of high SWNT content on the room temperature mechanical properties of fully dense 3YTZP/SWNTcomposites, J. Eur. Ceram. Soc. 34 (2014) 1571-1579.

${ }^{20}$ A. Morales-Rodríguez, A. Gallardo-López, A. Fernández-Serrano, R. Poyato, A. Muñoz, A. Domínguez-Rodríguez, Improvement of Vickers hardnessmeasurement on $\mathrm{SWNT} / \mathrm{Al}_{2} \mathrm{O}_{3}$ composites consolidated by spark plasma sintering, J. Eur. Ceram. Soc. 34 (2014) 3801-3809.

${ }^{21}$ M. Castillo-Rodríguez, A. Muñoz, A. Morales-Rodríguez, R. Poyato, A. GallardoLópez, A. Domínguez-Rodríguez, Influence of the processing route on the carbon nanotubes dispersion and creep resistance of 3YTZP/SWCNTs nanocomposites, J. Am. Ceram. Soc. 98 (2015) 645-53. 
${ }^{22}$ A. Gallardo-López, A. Morales-Rodríguez, J. Vega-Padillo, R. Poyato, A. Muñoz, A. Domínguez-Rodríguez, 3Y-TZP with fully dispersed SWNTs. Keys to processing and room temperature mechanical properties (in progress).

${ }^{23}$ M.H. Bocanegra-Bernal, A. Reyes-Rojas, A. Aguilar-Elguezabal, E. Torres-Moye, J. Echeberria, X-ray diffraction evidence of a phase transformation in zirconia by the presence of graphite and carbon nanotubes in zirconia toughened alumina composites, Int. J. Refrac. Metals Hard Mater. 35 (2012) 315-318.

${ }^{24}$ S. Rul, F. Lefèvre-Schlick, E. Capria, Ch. Laurent, A. Peigney, Percolation of singlewalled carbon nanotubes in ceramic matrix nanocomposites, Acta Mater. 52 (2004) 1061-1067.

${ }^{25}$ M.P. Garret, I.N. Ivanov, R.A. Gerhardt, A.A. Puretzky, D.B. Geohegan, Separation of junction and bundles resistance in single wall carbon nanotube percolation networks by impedance spectroscopy, Appl. Phys. Lett. 97 (2010) 163105.

${ }^{26}$ R.L. Muhlbauer, T.L. Pruyn, W.T. Puckett, R.A. Gerhardt, Effect of graphitic filler size and shape on the microstructure, electrical percolation behavior and thermal properties of nanostructured multilayered carbon films deposited onto paper substrates, J. Mater. Res. 29 (2014) 472-484.

${ }^{27}$ S.G. Louie, Electronic properties, junctions, and defects of carbon nanotubes. In: Dresselhaus MS, Dresselhaus G, Avouris P, editors. Carbon nanotubes: synthesis, structure, properties, and applications, vol. 80. Berlin: Springer; 2001. p. 113.

${ }^{28}$ P.N. Nirmalraj, P.E. Lyons, S. De, J.N. Coleman, J.J. Boland, Electrical connectivity in single-walled carbon nanotube networks, Nano Lett. 9 (2009) 3890-3895. 
${ }^{29}$ C. Li, E.T. Thostenson, T.W. Chou, Dominant role of tunneling resistance in the electrical conductivity of carbon nanotube-based composites, Appl. Phys. Lett. 91 (2007) 223114.

${ }^{30} \mathrm{P}$. Sheng, Fluctuation-induced tunneling conduction in disordered materials, Phys Rev B 21 (1980) 2180-2195.

${ }^{31}$ G. Bernard-Granger, C. Guizard, S. Surblé, G. Baldinozzi, A. Addad, Spark plasma sintering of a commercially available granulated zirconia powder II. Microstructure after sintering and ionic conductivity, Acta Mater. 56 (2008) 4658-4672. 